\title{
The end points in the dispersion of Holstein polarons
}

\author{
Glen L. Goodvin and Mona Berciu \\ Department of Physics 83 Astronomy, University of British Columbia, Vancouver, BC, Canada V6T $1 Z 1$
}

PACS 71.38.-k - Polarons and electron-phonon interactions

PACS 72.10.Di - Scattering by phonons, magnons, and other nonlocalized excitations

PACS 63.20.kd - Phonon-electron interactions

\begin{abstract}
We investigate the existence of end points in the dispersion of Holstein polarons in various dimensions, using the Momentum Average approximation which has proved to be very accurate for this model. An end point separates momenta for which the lowest-energy state is a discrete level, i.e., an infinitely-lived polaron, from those where the lowest-energy feature is a continuum in which the "polaron" is signalled by a resonance with a finite lifetime. While such end points are known to not appear in $1 \mathrm{D}$, we show here that they are generic in $3 \mathrm{D}$ if the particleboson coupling is not too strong. The 2D case is "critical": a pure 2D Holstein model has no end points, like the 1D case. However, any amount of interlayer hopping leads to 3D-like behavior. As a result, such end points are expected to appear in the spectra of layered, quasi-2D systems described by Holstein models. Generalizations to other models are also briefly discussed.
\end{abstract}

Introduction. - Understanding the properties of a novel material is inherently linked to understanding the physics of its underlying quasi-particles. The formation of such quasi-particles is a very old problem that comes up again and again in condensed matter physics. In particular, the formation of a quasi-particle $q p$ due to the coupling of the particle to bosonic degrees of freedom is relevant in many systems of current interest, such as manganites, cuprates, most other oxides, various organic materials, etc [1-4]. This coupling can be to phonons, magnons, orbitons, or any combination thereof, and the resulting composite object is known as a polaron.

Such polaron problems continue to attract considerable analytical and numerical interest, particularly for simple lattice Hamiltonians like the Holstein model [5] which can be studied by many means [6]. The ground-state is a discrete eigenstate, i.e., it describes a true quasiparticle with an infinite lifetime. At higher energies, however, the eigenstates may describe incoherent scattering of the particle on bosonic excitations, and have finite lifetimes.

One important question which has not been answered for lattice models of polarons, and which we settle for the Holstein polaron in this Letter, is whether the lowest eigenstate for a given momentum is an infinitely-lived, discrete state throughout the Brillouin zone, or not. That such a state may not exist for all momenta has long been suspected, since the dispersion of the Fröhlich polaron exhibits a so-called end point [7]. While this is a three- dimensional, continuous model, it is interesting to see if similar phenomenology is possible for lattice models, and under what conditions.

As we show below, the answer depends strongly on both the dimensionality of the system, and the value of the effective particle-boson coupling. At first sight, the former seems surprising, since many authors have demonstrated that dimensionality plays a very small role in determining the qualitative features of the ground state properties of the Holstein polaron [8-10]. However, the bare electron and polaron densities of states vary significantly with dimension [11], and as we show below, this plays an essential role in deciding whether a discrete level is pushed below the polaron+one-phonon continuum, or not. This continuum appears at $\Omega$ (the energy of the boson, modelled as an Einstein mode) above the ground-state energy, and describes excited states with a boson far from the polaron.

That the effective coupling is also very important is, on the other hand, not surprising. At very large couplings, the polaron effective mass is exponentially large, and therefore the polaron dispersion is very flat. As a result, one expects that the entire polaron band fits below the continuum, i.e., a true quasiparticle state exists at low energies in the entire Brillouin zone. At weaker couplings, however, the polaron dispersion is considerable and its band may overlap with the continuum at larger momenta. For the most studied case - the 1D chain - it is well known that irrespective of the coupling, (at least) one dis- 
crete state is pushed below the continuum everywhere in the Brillouin zone, although it flattens out just underneath the continuum at larger momenta. While it seems to be assumed in the literature that this is also the case in higher dimensions, we show here that this is not true in 3D. Indeed, in 3D at weak couplings, an infinitely lived polaron exists only in a finite region near the $\Gamma$ point. The $2 \mathrm{D}$ case is marginal: while a low-energy discrete state always exists in a purely $2 \mathrm{D}$ model, any amount of anisotropic hopping in the 3rd direction renders the system effectively 3D-like, and the transition from a true quasiparticle to a resonance becomes possible if the coupling is not too strong. In other words, unlike 1D systems, layered quasi-2D systems are not guaranteed to have a long-lived quasiparticle as the low-energy eigenstate throughout the Brillouin zone.

Formalism. - Our results are based on the Momentum Average (MA) approximation, initially developed to study the properties of the Holstein model [10] and then extended to more complex polaron models [12]. The approximation allows one to sum all diagrams appearing in the expansion of the self-energy, albeit with exponentially small contributions discarded so that this sum can actually be performed analytically. The MA approximation has been shown to be accurate over all of parameter space (except the extremely adiabatic limit), in all dimensions, and for all energies. Furthermore, the approximation satisfies the first six spectral weight sum rules exactly, and all higher order sum rules to a good degree of accuracy. It can also be systematically improved by including additional states, allowing for the correct reproduction of the polaron+one-phonon continuum. These improvements also lead to increased accuracy throughout parameter space, and exact agreement with additional spectral weight sum rules. Recently, the MA approximation has also been generalized to treat broken translational symmetry, due to disorder or surfaces [13], and also to obtain two-particle Green's functions needed for a calculation of the optical conductivity [14]. In conclusion, the MA approximation is a powerful and well-understood tool that allows us to understand very accurately the Holstein polaron physics, throughout the parameter space, for all energies and momenta. Also, because it is an analytical approximation, we can easily probe large regions of parameters space that are difficult for numerical techniques to investigate, especially in higher dimensions.

The Holstein model that we analyze here describes the simplest possible electron-phonon coupling on a lattice, and is given by [5]:

$\mathcal{H}=\sum_{\mathbf{k}}\left(\varepsilon_{\mathbf{k}} c_{\mathbf{k}}^{\dagger} c_{\mathbf{k}}+\Omega b_{\mathbf{k}}^{\dagger} b_{\mathbf{k}}\right)+\frac{g}{\sqrt{N}} \sum_{\mathbf{k}, \mathbf{q}} c_{\mathbf{k}-\mathbf{q}}^{\dagger} c_{\mathbf{k}}\left(b_{\mathbf{q}}^{\dagger}+b_{-\mathbf{q}}\right)$.

The first term is the kinetic energy of the free electron, with $c_{\mathbf{k}}^{\dagger}$ being electron creation operators for a state with momentum $\mathbf{k}$ (the spin is irrelevant in this model, and we ignore it in the following). For the free electron dispersion, we use nearest-neighbor hopping on a $d$-dimensional simple hypercubic lattice of lattice constant $a$ :

$$
\varepsilon_{\mathbf{k}}=-2 t \sum_{i=1}^{d} \cos \left(k_{i} a\right) .
$$

The coupling is to a branch of Einstein optical phonons of frequency $\Omega$, where $b_{\mathbf{k}}^{\dagger}$ and $b_{\mathbf{k}}$ are the phonon creation and annihilation operators. The last term describes a momentum-independent on-site linear coupling $g \sum_{i} c_{i}^{\dagger} c_{i}\left(b_{i}^{\dagger}+b_{i}\right)$, written in $\mathbf{k}$-space. All sums over momenta are over the first Brillouin zone. For all results shown here, the total number of sites $N$ is taken to be infinity. We also set $\hbar=1$ and $a=1$ throughout.

The MA approximation has been applied to the Holstein model previously, and its meaning and accuracy is very well understood [10]. Although the $\mathrm{MA}^{(0)}$ level of approximation has been shown to be quite accurate at describing ground state properties, it fails to reproduce the correct location for the polaron+one-phonon continuum. At the $\mathrm{MA}^{(1)}$ level and beyond, this continuum is accurately reproduced. Although the discussion and conclusions below do not qualitatively depend on the precise location of this continuum, we will employ the more accurate $\mathrm{MA}^{(1)}$ approximation throughout this work so that our results are quantitatively, not just qualitatively, accurate.

Application of the MA approximation to the Holstein Hamiltonian allows us to calculate the single-particle (retarded) Green's function, defined as $G(\mathbf{k}, \omega)=\langle 0| c_{\mathbf{k}}(\omega-$ $\mathcal{H}+i \eta)^{-1} c_{\mathbf{k}}^{\dagger}|0\rangle$ [15]. The solution for this Green's function can be written in the standard form [10]:

$$
G(\mathbf{k}, \omega)=\frac{1}{\omega-\varepsilon_{\mathbf{k}}-\Sigma_{\mathrm{MA}^{(n)}}(\mathbf{k}, \omega)+i \eta},
$$

with the $\mathrm{MA}^{(1)}$ self-energy given by:

$$
\Sigma_{\mathrm{MA}^{(1)}}(\omega)=\frac{g^{2} \bar{g}_{0}(\tilde{\omega})}{1-g \bar{g}_{0}(\tilde{\omega})\left[A_{2}(\omega)-A_{1}(\omega-\Omega)\right]},
$$

where

$$
\bar{g}_{0}(\omega)=\frac{1}{N} \sum_{\mathbf{k}} G_{0}(\mathbf{k}, \omega)
$$

is the momentum average over the Brillouin zone of the free propagator $G_{0}(\mathbf{k}, \omega)=\left(\omega-\varepsilon_{\mathbf{k}}+i \eta\right)^{-1}$. The functions $A_{n}(\omega)$ are infinite continued fractions, defined as:

$$
\begin{aligned}
A_{n}(\omega) & =\frac{n g \bar{g}_{0}(\omega-n \Omega)}{1-g \bar{g}_{0}(\omega-n \Omega) A_{n+1}(\omega)} \\
& =\frac{n g \bar{g}_{0}(\omega-n \Omega)}{1-\frac{(n+1) g^{2} \bar{g}_{0}(\omega-n \Omega) \bar{g}_{0}(\omega-(n+1) \Omega)}{1-\cdots}} .
\end{aligned}
$$

We have also used the short-hand notation $\tilde{\omega}=\omega-\Omega-$ $g A_{1}(\omega-\Omega)$. As discussed at length elsewhere [10], this MA self-energy is $\mathbf{k}$-independent simply because the Holstein model is so featureless. Indeed, momentum dependence for $\Sigma(\mathbf{k}, \omega)$ is obtained for the Holstein model for $\mathrm{MA}^{(n)}$ 
with $n \geq 2$ (although it is very weak). For other models with momentum-dependent couplings, a k-dependent self-energy is found even at the MA ${ }^{(0)}$ level [12]. Further details on the derivation and meaning of the MA approximation can be found in Ref. [10].

With an explicit expression for the Green's function of the Holstein polaron, we calculate the spectral weight

$$
A(\mathbf{k}, \omega)=-\frac{1}{\pi} \operatorname{Im} G(\mathbf{k}, \omega)
$$

from which we can extract the polaron dispersion, average number of phonons, effective mass, etc. [10]. In our calculations we employ a small but finite value for $\eta$. This moves the poles of the Green's function off of the real axis and changes the $\delta$-peaks of the spectral weight $A(\mathbf{k}, \omega)=\sum_{\alpha}\left|\left\langle\alpha\left|c_{\mathbf{k}}^{\dagger}\right| 0\right\rangle\right|^{2} \delta\left(\omega-E_{\alpha}\right)$ into Lorentzians. In practice, it is necessary to choose $\eta$ small enough to allow detection of the Lorentzian peaks in regimes where the $q p$ weight is extremely small. It is also convenient to define the dimensionless coupling constant $\lambda=g^{2} /(2 d \Omega t)$. We now turn our attention to the conditions that lead to the formation of an infinitely-lived polaron.

Results. - Separating the self-energy in terms of its real and imaginary parts, $\Sigma(\omega)=\Sigma^{\prime}(\omega)+i \Sigma^{\prime \prime}(\omega)$, we can write the spectral function in the following form:

$$
A(\mathbf{k}, \omega)=\frac{1}{\pi} \frac{\eta-\Sigma^{\prime \prime}(\omega)}{\left[\omega-\varepsilon_{\mathbf{k}}-\Sigma^{\prime}(\omega)\right]^{2}+\left[\eta-\Sigma^{\prime \prime}(\omega)\right]^{2}} .
$$

We are interested in the dispersion of the polaron, $E_{\mathbf{k}}$. This can be found by tracking the lowest pole in the spectral weight [10], its energy being the smallest value of $\omega$ which satisfies

$$
\omega-\varepsilon_{\mathbf{k}}=\Sigma^{\prime}(\omega) .
$$

For this to also be a discrete eigenstate (as opposed to the lower edge of a continuum), the imaginary part of the self-energy has to vanish:

$$
\left.\Sigma^{\prime \prime}(\omega)\right|_{\omega=E_{\mathbf{k}}}=0 .
$$

The latter requirement is essential, as eq. (7) does not have singular behavior when the imaginary part of the self-energy is finite. If eq. (9) is satisfied, we call the solution a true polaron $q p$, meaning that it has an infinite lifetime. If eq. (9) is not satisfied, then the lowest-energy feature is not a discrete state but the polaron+one-phonon continuum. In this case, we find the energy where this continuum has a maximum in the density of states (DOS), and call it a polaron resonance. This resonance has a finite lifetime proportional to $1 / \Sigma^{\prime \prime}(\omega)$, where $\omega$ is its energy.

As already mentioned, the onset of the continuum is at $E_{0}+\Omega$, where $E_{0}$ is the ground state energy of the polaron for $\mathbf{k}=0$ [10], therefore for $\omega<E_{0}+\Omega$ it is guaranteed that $\Sigma^{\prime \prime}(\omega)=0$. To summarize, we thus look for the region(s) of parameter space where $E_{\mathbf{k}}$, the lowestenergy solution of eq. (8), satisfies $E_{\mathbf{k}}<E_{0}+\Omega$. If this condition is satisfied, we have a true polaron bound state
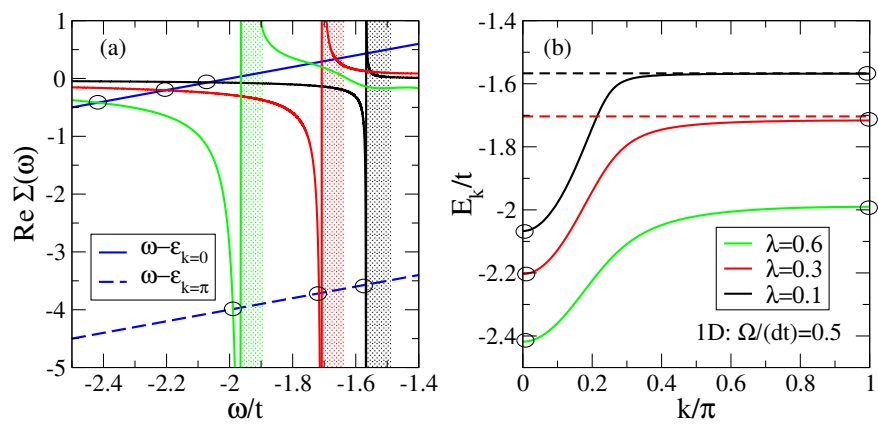

Fig. 1: (Color online) (a) The real part of the 1D self-energy $\Sigma(\omega)$ versus $\omega / t$, and (b) the polaron dispersion $E_{k}$, for effective el-ph coupling strengths $\lambda=0.1,0.3$ and 0.6. In (a) we also plot $\omega-\varepsilon_{k}$, for $k=0$ (solid blue line) and $k=\pi$ (dashed blue line). The intersection of these curves with $\operatorname{Re} \Sigma(\omega)$ give the polaron energy eigenvalues, shown by the respective circles in (a) and (b). The shaded areas indicate the onset of the continuum, i.e., where $\operatorname{Im} \Sigma(\omega) \neq 0$.

at that momentum. Otherwise, we have a polaron-like resonance with a finite lifetime lying inside the continuum, as described above.

Obviously, it is the self-energy that controls which of the two possible cases is encountered. To understand how this occurs, we first quickly analyze the well-known 1D case, where - as already mentioned - there is always a true polaron $q p$ state below the continuum. The reason for this is made obvious by fig. 1(a), where we show a geometric solution of eq. (8) for three different values of the effective coupling $\lambda$. The real part of the self-energy, $\Sigma^{\prime}(\omega)$, goes asymptotically like $\frac{g^{2}}{\omega}$ as $\omega \rightarrow-\infty$, so it starts as a negative function which, in $1 \mathrm{D}$, diverges and changes sign at a value marking the onset of the polaron+one-phonon continuum (above this energy, in the shaded regions, $\Sigma^{\prime \prime}(\omega)$ becomes finite). The left-hand side of eq. (8) is a straight line - the limiting $k=0$ and $k=\pi$ are drawn explicitly and a true polaron solution exists if the two intersect below the onset of the continuum. The divergence of $\Sigma^{\prime}(\omega)$ ensures that in $1 \mathrm{D}$ there is always such a solution. Its energy $E_{\mathbf{k}}$ is shown in fig. 1(b), where we also plotted the onset of the continuum, $E_{0}+\Omega$, for the three cases.

As expected, at very weak couplings and sufficiently large $\mathbf{k}, E_{\mathbf{k}}$ flattens out just below the continuum. Geometrically, we see that this is due to the fact that for a large region of the Brillouin zone, the intersection between the two curves occurs very close to the divergence in $\Sigma^{\prime}(\omega)$, so the value of the solution $\omega$ varies little and is asymptotically close to $E_{0}+\Omega$. The value of $k$ where this "flattening" begins is also easy to estimate for $\lambda \rightarrow 0$ : in this limit, to zero order in perturbation theory we must have $E_{\mathbf{k}} \approx \varepsilon_{\mathbf{k}}$, therefore the "flattening" must happen for momenta $\mathbf{k}$ such that $\varepsilon_{\mathbf{k}}>\varepsilon_{\mathbf{0}}+\Omega$. Here, because of the divergence in the free-electron DOS at the band-edge, arbitrarily weak coupling to the phonons suffices to repel a bound state below the continuum. As the coupling $\lambda$ increases, the self-energy curve shifts to lower energies, but 

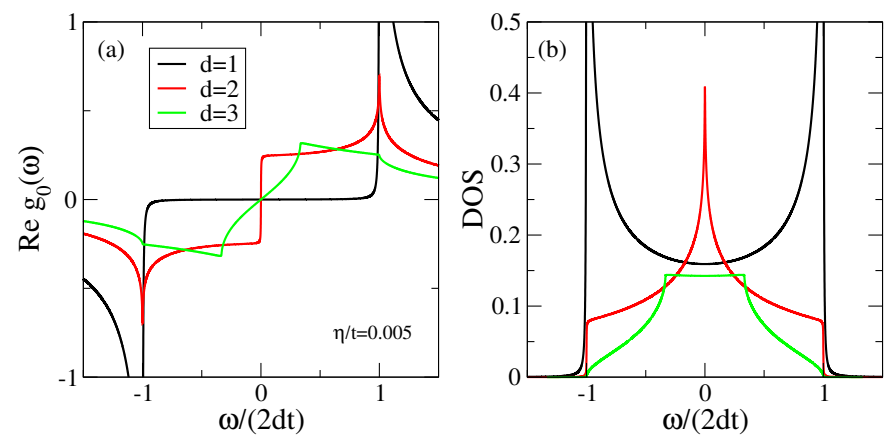

Fig. 2: (Color online) (a) The real part of $\bar{g}_{0}(\omega)$ and (b) the bare electron $\operatorname{DOS}(-1 / \pi) \operatorname{Im} \bar{g}_{0}(\omega)$, shown for $d=1,2,3$. A broadening factor of $\eta / t=0.005$ has been used.

the divergence is always there.

Clearly, the next step is to understand why the selfenergy has this shape and in particular the reason for the divergence in its real part. If such a divergence is always guaranteed to mark the onset of the continuum, then a discrete polaron state will always be found.

As already discussed, we are actually primarily interested in the limit of weak el-ph coupling, since at large el-ph coupling the polaron band is very flat and guaranteed to obey $E_{\mathbf{k}}<E_{0}+\Omega$ everywhere in the Brillouin zone. For weak coupling $g \rightarrow 0$, eq. (3) gives a self-energy

$$
\Sigma(\omega) \approx g^{2} \bar{g}_{0}(\omega-\Omega)+\cdots=\frac{g^{2}}{N} \sum_{\mathbf{k}} G_{0}(\mathbf{k}, \omega-\Omega)+\cdots
$$

after using eq. (4). We recognize this as being the lowest order diagram contributing to the self-energy (the Born approximation), in agreement with perturbation theory. The correction $\omega-\Omega \rightarrow \tilde{\omega}$ in the argument of $\bar{g}_{0}(\omega)$ will further shift the self-energy towards lower energies, and is due to corrections from higher order diagrams. However, this shift cannot be responsible for the appearance of a singularity in the real part of the self-energy.

It follows that for the Holstein model (we discuss other models below) the real part of $\bar{g}_{0}(\omega)$ controls the energy dependence of $\Sigma^{\prime}(\omega)$ for small $\lambda$, and therefore whether it has a singularity or not. Both the real and imaginary parts of $\bar{g}_{0}(\omega)$ are shown for various dimensions in fig. 2. Actually, the right panel shows $(-1 / \pi) \operatorname{Im} \bar{g}_{0}(\omega)$, which from eq. (4) is seen to be the bare electron total DOS.

Because $\bar{g}_{0}(\omega)$ is the momentum average of a retarded propagator, its real and imaginary parts are related through Kramers-Kronig relations. Given that the imaginary part (the DOS) is finite only inside the free-electron bandwidth $|\omega| \leq 2 d t$, it follows that it is the DOS at the band-edge that controls whether the real part has a singularity at the band-edge energy: if the band-edge DOS diverges, or at least has a discontinuous jump, then the real part has a singularity at the band-edge; otherwise it is finite everywhere.

This explains why in 1D we always find a singularity in the real part of $\bar{g}_{0}(\omega)$, and therefore in $\Sigma^{\prime}(\omega)$ : it is well-
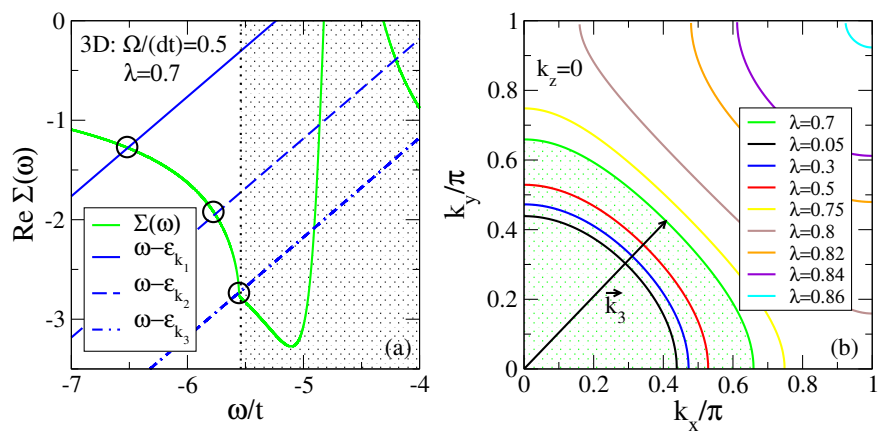

Fig. 3: (Color online) (a) The real part of the 3D self-energy for an intermediate el-ph coupling $\lambda=0.7$. The shaded region shows where $\operatorname{Im} \Sigma(\omega) \neq 0$. The straight lines show $\omega-\varepsilon_{\mathbf{k}}$ curves for $\mathbf{k}_{1}=\pi(0.2,0.2,0), \mathbf{k}_{2}=\pi(0.3,0.3,0), \mathbf{k}_{3}=\pi(0.43,0.43,0)$. An intersection of $\omega-\varepsilon_{\mathbf{k}}$ and $\operatorname{Re} \Sigma(\omega)$ corresponds to a solution of eq. (8); (b) Contours of the end points for various el-ph coupling strengths. The $\lambda=0.7$ contour is shaded, and the arrow marks the end point $\mathbf{k}_{c}=\mathbf{k}_{3}$ found in (a).

known that the free electron DOS for nearest-neighbor hopping in 1D has van Hove singularities at the band edges. In $2 \mathrm{D}$, the DOS has discontinuities at the band edges. As a result, based on the discussion above, we expect that in $2 \mathrm{D}$ a discrete polaron state must also exist in the entire Brillouin zone, just like in 1D. However, this is a very weak logarithmic singularity (as it is due to a discontinuity, not a singularity in the DOS) and one may expect that it can be easily removed. This is indeed the case, as we show below where we analyze quasi-2D systems.

However, first we focus on the 3D case, where the DOS at the band-edge is continuous, leading to a real part of $\bar{g}_{0}(\omega)$, and therefore low-coupling $\Sigma(\omega)=g^{2} \bar{g}_{0}(\tilde{\omega})$, that is finite everywhere. The geometric solution of eq. (8) in this case is illustrated in the left panel of fig. 3, for an intermediate effective coupling $\lambda=0.7$. The low-energy part of $\Sigma^{\prime}(\omega)$ still resembles the real part of $\bar{g}_{0}(\omega)$, although due to higher order diagrams, the shape is somewhat distorted. The differences are more considerable at higher energies (this issue is revisited below). The onset of the continuum at $E_{0}+\Omega$ is marked by a discontinuity in the slope of $\Sigma^{\prime}(\omega)$ (shaded area). Because $\Sigma^{\prime}(\omega)$ does not diverge below this energy, it is now apparent that eq. (8) only has solutions with $E_{\mathbf{k}}<E_{0}+\Omega$ up to a critical value $\mathbf{k}_{c}$ which marks the "end point" in the polaron dispersion [7]. For $\lambda=0.7$ and along the $(1,1,0)$ direction, the critical value is at $\mathbf{k}_{c}=\pi(0.43,0.43,0)$, as shown in fig. 3(a).

Repeating this along various directions allows us to identify the surface described by the "end points" $\mathbf{k}_{c}$ in the Brillouin zone, which marks the separation between the region near the $\Gamma$ point where an infinitely-lived quasiparticle exists, and the region near the edges of the Brillouin zone where only a resonance with a finite lifetime appears. The end points contours in a quadrant of the $k_{z}=0$ plane are shown in fig. 3(b), for various values of $\lambda$. The arrow marks the particular $\mathbf{k}_{c}$ found in fig. 3(a).

For a given value of the phonon mode, the smallest sur- 

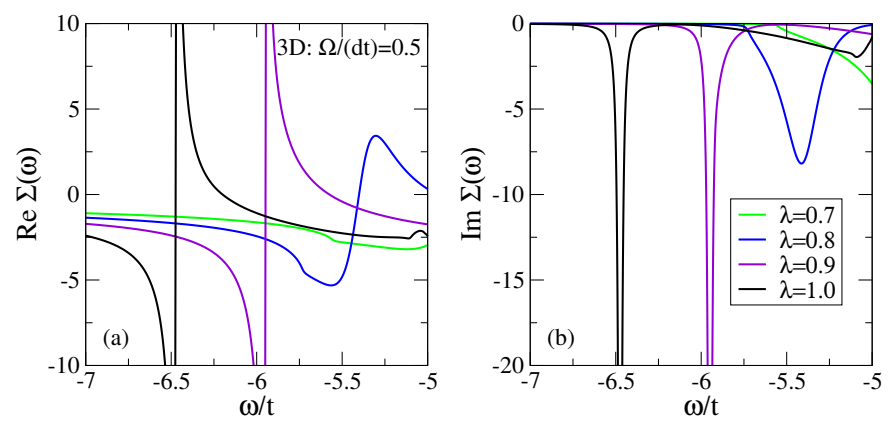

Fig. 4: (Color online) The (a) real and (b) imaginary parts of the 3D self-energy, for increasing el-ph coupling strengths. The self-energy becomes divergent for large enough coupling.

face of end points is for $\lambda \rightarrow 0$. By analogy with a previous discussion, in this case we can estimate it to be given by $\varepsilon_{\mathbf{k}_{c}} \approx \varepsilon_{0}+\Omega$, since for $\lambda \rightarrow 0, E_{\mathbf{k}} \approx \varepsilon_{\mathbf{k}}$. It follows that for any $\Omega>0$, there is a vicinity of the $\Gamma$ point where a true polaron exists. In general, however, there are regions in the Brillouin zone that do not satisfy this condition for small $\lambda$. Indeed, since the maximum value of $\varepsilon_{\mathbf{k}}$ is $6 t$ while $\varepsilon_{0}=-6 t$, we estimate that for any $\Omega<12 t$ there exist such regions of momenta without an infinitely lived polaron state. $\Omega<12 t$ is likely to hold in most materials, therefore such a case is rather typical. If $\Omega$ is not too large, then in the limit $\lambda \rightarrow 0$ the surface of end points is spherical, since at low energies the free-electron dispersion can be approximated by a parabola. As either $\Omega$ or $\lambda$ increase, the shape will expand and distort, as seen in fig. 3(b) for increasing $\lambda$. For any value of $\Omega<12 t$, there is a critical value $\lambda_{c}>0$ above which an infinitely-lived polaron solution appears in the entire Brillouin zone. For $\Omega=0.5 t$, fig. 3(b) reveals that this $\lambda_{c}$ is just above 0.86 .

As already discussed, the fact that a true polaron solution exists everywhere in the Brillouin zone at strong(er) coupling is not surprising, because the significant increase in the polaron effective mass guarantees that eventually the whole polaron band will fit below the continuum. In terms of the geometrical solution, what happens is revealed in fig. 4 which shows that for large enough $\lambda$, the real part of the self-energy does eventually gain a singularity at low-energies. This divergence is a higher order effect, not coming from a divergence/discontinuity in the DOS, but due to the structure of the self-energy itself.

Eq. (3) reveals that at large enough el-ph coupling $g$, the denominator itself can vanish giving rise to a different type of divergence in the self-energy. The appearance of this divergence explains the phenomenology related to $\lambda_{c}$ (for $\Omega=0.5 t$, this divergence is first observed at $\lambda_{c}=$ 0.87). Physically, it is linked to the formation of the second bound state $[10,16]$, which is known to form below the polaron+one-phonon continuum as the crossover towards the small polaron regime is approached for $\lambda \sim 1$.

For completeness, we mention that as the coupling increases further, more and more divergences appear in $\Sigma^{\prime}(\omega)$ due to the structure of the continuous fractions, see
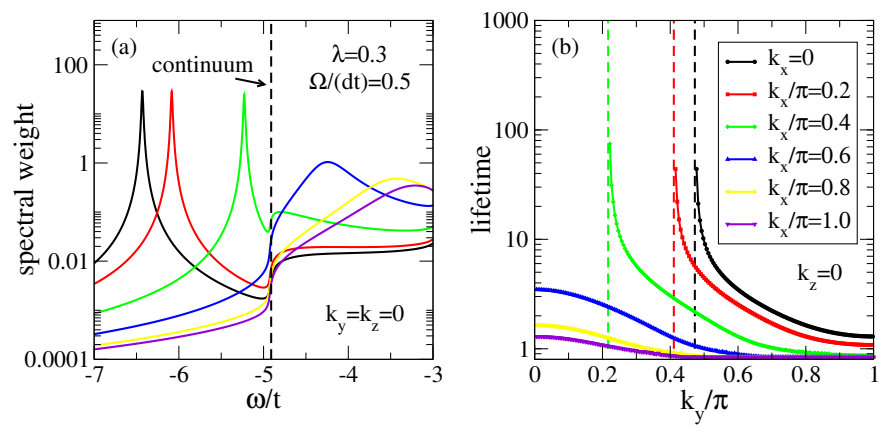

Fig. 5: (Color online) (a) The spectral weight $A(\mathbf{k}, \omega)$ versus $\omega$ with $k_{y}=k_{z}=0$, and $k_{x} / \pi=0,0.2,0.4,0.6,0.8,1.0$. The dashed line denotes the onset of the continuum. With the parameters shown, there is no longer a polaron $q p$ for $k_{x} / \pi>$ 0.46 , but a polaron-like resonance. (b) The lifetime of the polaron-like resonance as a function of momentum. The dashed lines denote the transition to an infinitely lived polaron state.

eq. (5). These additional divergences occur at higher and higher energies that are well above the onset of the continuum, thus they are not relevant for the problem we consider here (they are linked to the crossover towards a LangFirsov type of spectrum expected in the limit $\lambda \rightarrow \infty$ ).

What happens for $\lambda<\lambda_{c}$ as we sweep through $\mathbf{k}_{c}$ is shown in fig. 5(a), where we plot the spectral weight $A(\mathbf{k}, \omega)$ vs. $\omega$, for several values of $\mathbf{k}$. The onset of the continuum is marked by the dashed vertical line. For momenta below $\mathbf{k}_{c}$, we see a Lorentzian peak below the continuum. Its width is controlled by $\eta$. This is the discrete level whose energy is $E_{\mathbf{k}}$. As $\mathbf{k}$ increases, $E_{\mathbf{k}}$ approaches the continuum. Unlike in 1D and 2D cases, however, where the singularity/discontinuity in the DOS ensured that a discrete level is always pushed below the continuum, here we see that above $\mathbf{k}_{c}$, the low-energy feature is the continuum, with a broad maximum that disperses towards higher energies while broadening even more as $\mathbf{k}$ increases - this is the polaron resonance. Its finite lifetime, equal to $1 / \Sigma^{\prime \prime}(\omega)$ where $\omega$ is its energy, is displayed in fig. $5(\mathrm{~b})$ along various cuts in the Brillouin zone. As expected, it diverges as $\mathbf{k}_{c}$ is approached from above, and it decreases very fast towards the edges of the Brillouin zone. This is a $T=0$ calculation, so this finite lifetime is an intrinsic effect. As a technical note, in calculating it we decrease $\eta$ until changing it any further has no effect on the lifetime.

Based on all these results, we can now quickly analyze the 2D case. For a purely planar 2D hopping model, the well-known discontinuity in the DOS at the band-edge guarantees that a low-energy singularity always exists in $\Sigma^{\prime}(\omega)$, see fig. 2. As a consequence, a true polaron is guaranteed to form for all $\mathbf{k}$ at any $\lambda$, just like in 1D. However, most layered materials are in fact quasi-2D, because there is some small anisotropic hopping between different planes. The effect of a small $t_{z}=0.1 t$ on the $\bar{g}_{0}(\omega)$ function is shown in fig. 6. Any $t_{z} \neq 0$ will smooth out the band-edge discontinuity in the DOS, and thus remove the weak singularity in the real part of $\bar{g}_{0}(\omega)$ and therefore 

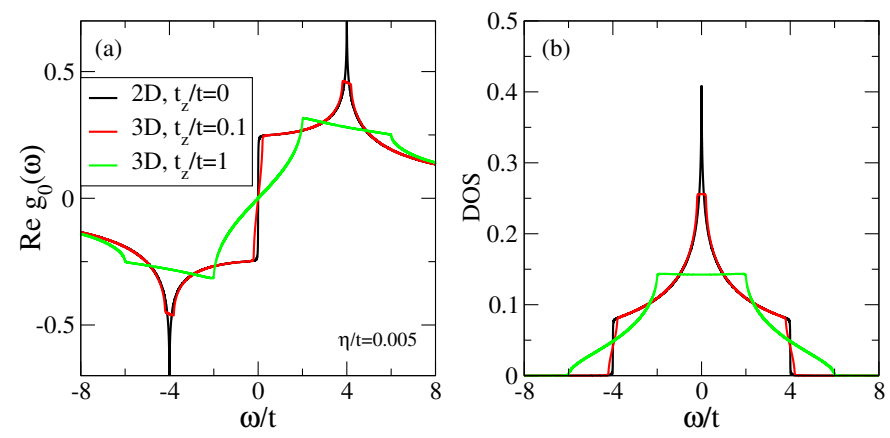

Fig. 6: (Color online) Anisotropic hopping - transition from $2 \mathrm{D}$ to $3 \mathrm{D}$ DOS.

in $\Sigma^{\prime}(\omega)$ at weak coupling. As a result, quasi-2D systems behave similarly to 3D systems: for weak-to-intermediate coupling, we expect that a surface of end points separate the region near $\Gamma$ where a true polaron quasiparticle forms, from a region near the Brillouin zone edges where only a finite lifetime resonance appears. The detailed shape of this surface and the critical couplings $\lambda_{c}$ above which a true polaron exists everywhere will, of course, depend on the particular parameters of the problem. However, the reasons we uncovered here to explain this phenomenology are robust and therefore such surfaces of end points should be quite generic in quasi-2D and $3 \mathrm{D}$ materials with not too large el-ph coupling.

The existence of such surfaces of end points, especially in layered systems, should be directly visible using Angle Resolved Photo-Emission Spectroscopy [17]. This technique measures the spectral weight and should be able to identify significant changes in its shape, like those shown in fig. 5(a). Very different temperature dependence of features in different regions of the Brillouin zone would also be consistent with such phenomenology, because the true $q p$ peaks should only exhibit thermal broadening, while the finite-lifetime resonances have an intrinsic lifetime. Of course, many other properties should be strongly influenced by the existence of such a surface of end points, especially if the Fermi surface is not too far from it (this statement assumes that interactions between polarons, either due to direct interactions between the particles or mediated through exchange of bosons between the two polaronic clouds, do not qualitatively change this phenomenology. Whether this is the case or not is a very interesting question which still awaits resolution).

To summarize, for a Holstein model and weak-tointermediate coupling one can deduce easily whether an infinite lifetime polaron $q p$ is expected everywhere in the Brillouin zone or not simply from knowing whether the DOS of the free electrons is continuous or not at the band-edge. To pinpoint the surface of end points, more detailed calculations like the ones shown here are needed. For more complicate polaron models, with a particle-boson coupling that depends on either (or both) the particle and boson momentum, and also with a possibly dispersing bo- son mode, one can certainly gain intuition about what is likely to happen from studying the Born approximation

$$
\Sigma(\mathbf{k}, \omega) \approx \frac{1}{N} \sum_{\mathbf{q}}\left|g_{\mathbf{k}, \mathbf{q}}\right|^{2} G_{0}\left(\mathbf{k}-\mathbf{q}, \omega-\Omega_{\mathbf{q}}\right)
$$

and whether it generically predicts infinitely-lived quasiparticles for all $\mathbf{k}$ or not (if there are no end points at weak coupling, they are very unlikely to appear at stronger couplings for reasons already discussed). If either $g_{\mathbf{k}, \mathbf{q}}$ or $\Omega_{\mathbf{q}}$ have strong momentum dependence, then $\Sigma(\mathbf{k}, \omega)$ may acquire a sufficiently strong $\mathbf{k}$-dependence to modify the conditions found above for the Holstein model. In particular, it is not apriorily obvious that a singularity in $\Sigma^{\prime}(\mathbf{k}, \omega)$ is absolutely needed to guarantee a discrete low-energy solution everywhere in the Brillouin zone, if its momentum is strong enough to shift the curve around significantly.

$$
* * *
$$

This work was supported by NSERC and CIfAR. We thank N. Prokof'ev for suggesting the problem, and G. A. A. Sawatzky for many useful discussions.

\section{REFERENCES}

[1] Salamon M.B. and Jaime M., Rev. Mod. Phys., 73 (2001) 583.

[2] Shen K.M. et al., Phys. Rev. Lett., 93 (2004) 267002.

[3] Hengsberger M., et al., Phys. Rev. Lett., 83 (1999) 592.

[4] Matsui H. et al., Phys. Rev. Lett., 104 (2010) 056602.

[5] Holstein T., Ann. Phys. (N.Y.), 8 (1959) 325.

[6] Alexandrov A.S. (Editor), Polarons in Advanced Materials (Canopus, Bath/Spring-Verlag, Bath) 2007.

[7] Prokof'ev N.V. and Svistunov B.V., Phys. Rev. Lett., 81 (1998) 2514.

[8] Romero A.H., Brown D.W., and Lindenberg K., Phys. Rev. B, 60 (1999) 14080.

[9] Ku L.-C., Trugman S., and Bonca J., Phys. Rev. B, 65 (2002) 174306.

[10] Berciu M., Phys. Rev. Lett., 97 (2006) 036402; Goodvin G.L., Berciu M., and Sawatzky G.A., Phys. Rev. B, 74 (2006) 245104; Berciu M. and Goodvin G.L., Phys. Rev. B, 76 (2007) 165109.

[11] Kornilovich P.E., Phys. Rev. B, 60 (1999) 3237.

[12] Goodvin G.L. and Berciu M., Phys. Rev. B, 78 (2008) 235120; Berciu M. and Fehske H., (unpublished).

[13] Berciu M., Mishchenko A.S. and Nagaosa N., Euro. Phys. Lett., 89 (2010) 37007; Goodvin G.L., CovaCi L. and Berciu M., Phys. Rev. Lett., 103 (2009) 176402.

[14] Goodvin G.L. and Berciu M., (unpublished).

[15] Mahan G.D., Many particle physics (Plenum, New York) 1981.

[16] Bonca J., Trugman S. A. and Batistic I., Phys. Rev. $B, 60$ (1999) 1633.

[17] Damascelli A., Hussain Z., and Z.-X. Shen, Rev. Mod. Phys., 75 (2003) 473. 